\title{
New syndrome reconciles theories of ageing
}

DOI:

10.1038/nrg2050

URLs

Entrez gene:

ERCC1: http://www.ncbi.nlm. nih.gov/entrez/query.fcgi?db=g ene \&cmd=Retrieve $\&$ dopt $=$ full report\&list_uids $=2072$

XPF: http://www.ncbi.nlm.nih gov/entrez/query.fcgi? $\mathrm{db}=$ gen e\&cmd $=$ Retrieve\&dopt $=$ full report\&list_uids=7509

OMIM:

xeroderma pigmentosum http://www.ncbi.nlm.nih gov/entrez/dispomim. cgi?id $=278700$
Is ageing merely the result of the accumulation of cellular damage over time, or is it genetically regulated? A new premature-ageing syndrome and a comparison of the transcriptomes of naturally and prematurely aged mice reconcile these two theories. They indicate the existence of a programmed response to DNA damage accumulation that is mediated by the insulin-like growth factor (IGF) pathway and is associated with ageing.

Mutations in components of the nucleotide excision repair (NER) pathway can cause either premature ageing or cancer susceptibility. Premature ageing arises from mutations that affect the repair of lesions that block transcription; cancer susceptibility is caused by mutations that affect genome-wide mutation prevention, irrespective of transcrip- tion. The authors have identified a new syndrome - XPF-ERCC1 progeria (XFE) - that combines both phenotypes. XPF-ERCC1 is a two-subunit endonuclease that is required for NER. Patients with mild mutations in XPF have xeroderma pigmentosum, a cancer-susceptibility syndrome. However, a patient with a severe mutation in a highly conserved residue of the same gene also shows premature ageing.

To investigate further, the authors created a mouse model of this syndrome - an Ercc1-null strain. They compared the liver and kidney transcriptomes of young mutant mice with naturally aged wild-type mice, and also wild-type mice that were subjected to a genotoxic stress. All three showed similar differences in gene expression to the controls: growth-promoting

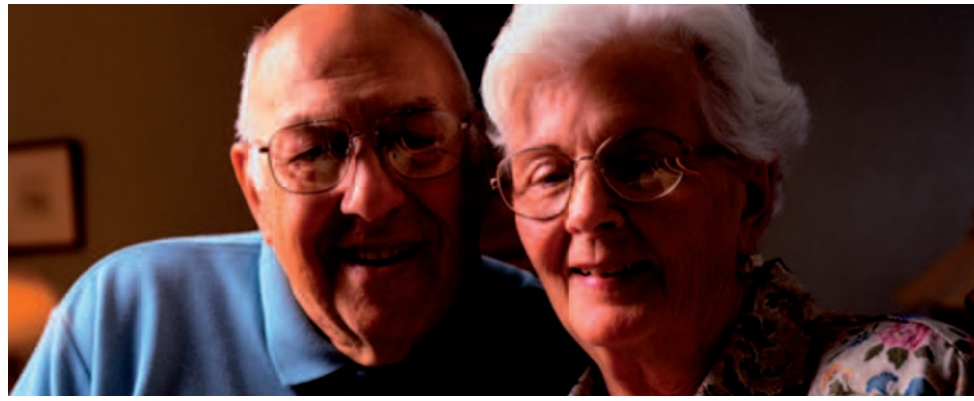

hormones, especially those in the IGF pathway, were downregulated, whereas DNA repair, anti-oxidant defence, glucose storage and apoptosis were upregulated.

The authors propose that increased DNA damage, as a result of natural ageing, environmental insult or genetic deficiency, triggers a switch from growth to repair and the prolonging of life. This is consistent with the effects of mutations in IGF pathway components and reduced oxidative damage through caloric restriction, both of which prolong life. This link between DNA repair and ageing shows that damage accumulation does cause ageing, but that its progress is tightly regulated by the IGF pathway and repair capacity. The next step is to elucidate the mechanism by which IGF does this.

Patrick Goymer

ORIGINAL RESEARCH PAPER Niedernhofer, L.

et al. A new progeroid syndrome reveals that genotoxic stress suppresses the somatotroph axis. Nature 444, 1038-1044 (2006)

FURTHER READING Capell, B. C. \& Collins, F. S. Human laminopathies: nuclei gone genetically awry. Nature Rev. Genet. 7, 940-952 (2006)| Christensen, K., Johnson, T. E. \& Vaupel, J. W. The quest for genetic determinants of human longevity: challenges and insights. Nature Rev. Genet. 7, 436-448 (2006) | Longo, V. D., Mitteldorf,

J. \& Skulachev, V. P. Programmed and altruistic ageing. Nature Rev. Genet. 6, 866-872 (2005) 